\title{
Hydrogen Evolution Reaction Activity of Heterogeneous Materials: A Theoretical Model
}

\author{
Joakim Ekspong, Eduardo Gracia-Espino,* and Thomas Wågberg* \\ Cite This: J. Phys. Chem. C 2020, 124, 20911-20921 \\ Read Online
}

ABSTRACT: In this study, we present a new comprehensive methodology to quantify the catalytic activity of heterogeneous materials for the hydrogen evolution reaction (HER) using ab initio simulations. The model is composed of two parts. First, the equilibrium hydrogen coverage is obtained by an iterative evaluation of the hydrogen adsorption free energies $\left(\Delta G_{\mathrm{H}}\right)$ using density functional theory calculations. Afterward, the $\Delta G_{\mathrm{H}}$ are used in a microkinetic model to provide detailed characterizations of the entire HER considering all three elementary steps, i.e., the discharge, atom + ion, and combination reactions, without any prior assumptions of rate-determining steps. The microkinetic model takes the equilibrium and potential-dependent character-
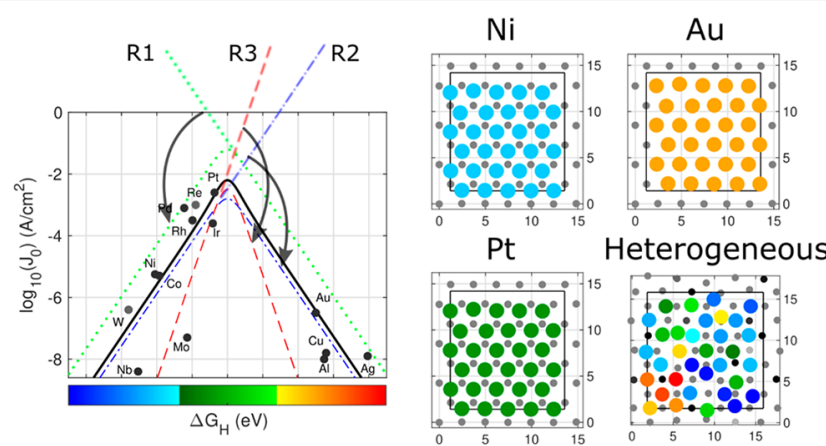
istics into account, and thus both exchange current densities and Tafel slopes are evaluated. The model is tested on several systems, from polycrystalline metals to heterogeneous molybdenum disulfide $\left(\mathrm{MoS}_{2}\right)$, and by comparing to experimental data, we verify that our model accurately predicts their experimental exchange current densities and Tafel slopes. Finally, we present an extended volcano plot that correlates the electrical current densities of each elementary reaction step to the coverage-dependent $\Delta G_{\mathrm{H}}$.

\section{INTRODUCTION}

The hydrogen evolution reaction (HER) is a fundamental electrochemical reaction occurring in water electrolysis to produce hydrogen. While it is possible to operate water electrolyzers with zero $\mathrm{CO}_{2}$ emissions, the hydrogen production costs using this method are still high compared to those of other technologies. ${ }^{1-4}$ Finding more active and abundant electrocatalysts for HER is therefore an important step in making water electrolysis a truly sustainable and efficient alternative. ${ }^{5,6}$ In the past decades, the design and development of electrocatalysts have benefitted greatly from having a deeper theoretical understanding. ${ }^{7,8}$ In the 1950s, Parsons established that the hydrogen adsorption free energy $\left(\Delta G_{H}\right)$ in metals could describe their catalytic activity toward HER with a so-called volcano-like relation, ${ }^{9}$ where an optimal adsorption energy and hence maximum activity are found at the top of the volcano with $\Delta G_{\mathrm{H}}=0 \mathrm{eV}$. Many other studies have described the electrochemical reaction rate with similar kinetics-based models. ${ }^{10-15}$ Nowadays, the $\Delta G_{\mathrm{H}}$ value can be rather easily calculated using density functional theory (DFT), as first described by Nørskov et al. in 2005, in which they further developed a simple kinetic model that is effective for homogeneous materials and gives the exchange current density under equilibrium conditions. ${ }^{16}$ More recent models have thereafter been developed in which the surface hydrogen coverage and solvent effects have been implemented. ${ }^{15,17,18}$ Nevertheless, the introduction of a realistic double-layer model with solvent effects is rather complicated and is therefore not frequently investigated. Furthermore, when studying heterogeneous materials containing different elements, defects, or other structural variations, a detailed model of the whole system is preferable. ${ }^{19,20}$ In many theoretical studies, however, it is common that only the adsorption sites displaying the highest activity are reported, even if heterogeneous materials are involved. Such a procedure does not necessarily render accurate representations of the total activity, hence there is poor agreement between theoretical and experimental data. Although several adsorption isotherms have been developed to handle heterogeneity, they are rarely implemented for HER using $a b$ initio data of $\Delta G_{\mathrm{H}} \cdot{ }^{21}$

Here, we propose an extensive theoretical model to characterize heterogeneous materials for HER using a bottomup approach. The current densities are calculated from $\Delta G_{\mathrm{H}}$ obtained under equilibrium conditions using DFT but also as a function of the overpotential allowing extensive characterizations, such as voltammetry plots. To calculate the current

Received: June 9, 2020

Revised: August 13, 2020

Published: August 17, 2020 
densities, all elementary reaction steps (both forward and backward) are included in the complete reaction, and each adsorption site is studied individually before the total activity of the material is determined. Therefore, the evolution of the ratelimiting step with an overpotential can be observed. Since we combine all adsorption sites to calculate the total activity, any atomic variations such as doping, vacancies, distortions, and strains in the material are included.

We carefully revised both the experimental and theoretical exchange current densities of several metals to evaluate the model. The obtained results agree well with the experimental data with regard to both exchange current densities and Tafel slopes. Finally, we test and verify the model successfully on a heterogeneous structure, representing a molybdenum disulfide catalyst containing various kinds of edge atoms together with a basal plane. Computer scripts for SIESTA and MATLAB of the described methodology are available online.

\section{METHODS}

All symbols used in the article are described in Table S1 in the Supporting Information.

2.1. Computational Details. Ab initio calculations were performed using density functional theory using the SIESTA code. $^{22,23}$ The generalized gradient approximation (GGA) with the revised Perdew, Burke, and Ernzerhof (RPBE) parametrization was used to describe the exchange and correlation functional. ${ }^{24}$ The valence electrons were represented by a linear combination of pseudoatomic numerical orbitals using a double$\zeta$ polarized basis. ${ }^{25}$ A mesh cutoff of 250 Ry was used for the realspace grid. The criterion for self-consistency in the selfconsistent-field cycle was $10^{-4} \mathrm{eV}$ for the maximum difference on each element in the density matrix for two consecutive steps. All systems were relaxed using a variable cell scheme by conjugate gradient minimization until the maximum forces were $<0.035 \mathrm{eV} / \AA$. For all unit cell optimizations, we used a Monkhorst-Pack k grid of $14 \times 14 \times 14,18 \times 18 \times 18$, and $15 \times 15 \times 8$ in the fcc, bcc, and hcp systems, respectively. Squared metal slabs were thereafter constructed with 4 atomic layers, each containing $18,16,16,12$, and 16 atoms for the fcc100, fcc111, bcc100, bcc110, and hcp0001 systems, respectively, with a vacuum of $20 \AA$ inserted in the $z$ direction to avoid interactions between neighboring simulation cells. A Monkhorst-Pack k grid of $2 \times 2 \times 1$ was used for the geometric relaxations of all metal slabs. ${ }^{26}$ Spin-polarized calculations were performed for Co and Ni. $\Delta G_{\mathrm{H}}$ was evaluated by using the computational hydrogen electrode (CHE) model. ${ }^{16} \Delta G_{\mathrm{H}}$ was calculated as $\Delta G_{\mathrm{H}}=\Delta E_{\mathrm{H}}+\Delta E_{\mathrm{ZPE}}-T \Delta S$, where $\Delta E_{\mathrm{H}}$ is the hydrogen adsorption energy evaluated using $\mathrm{H}_{2}(\mathrm{~g})$ as the reference state. The total energy of the $\mathrm{H}_{2}$ molecule in vacuum was calculated to be $-32.019 \mathrm{eV}$; therefore, the energy of a $\mathrm{H}$ atom is half this value according to the model. $T$ is the temperature $(298.15 \mathrm{~K})$, and $\Delta E_{\mathrm{ZPE}}$ and $\Delta S$ are the changes in the zero-point energy and entropy between the adsorbed and gas phases of a hydrogen atom. The term $\left(\Delta E_{\mathrm{ZPE}}-T \Delta S\right)$ has previously been calculated to be $+0.24 \pm 0.02 \mathrm{eV}$ for hydrogen adsorption on several metals. ${ }^{15,16,27}$ Therefore, we use $0.24 \mathrm{eV}$ in all calculations. During $\mathrm{H}$ adsorption, the bottom two layers of the metal slabs and the cell parameters were kept fixed while all other atoms were allowed to relax.

All microkinetic modeling was performed using MATLAB.

2.2. Hydrogen Evolution Reaction. The HER is examined under acidic conditions involving three elementary reaction steps, exemplified in R1-R3 and visualized in Figure 1.

$$
\begin{aligned}
& \text { discharge adsorption: } \mathrm{H}^{+}+\mathrm{e}^{-} \rightarrow \mathrm{H}_{\mathrm{ads}} \\
& \text { electrochemical desorption: } \mathrm{H}^{+}+\mathrm{e}^{-}+\mathrm{H}_{\mathrm{ads}} \rightarrow \mathrm{H}_{2}(\mathrm{~g})
\end{aligned}
$$

combination desorption: $\mathrm{H}_{\mathrm{ads}}+\mathrm{H}_{\mathrm{ads}} \rightarrow \mathrm{H}_{2}(\mathrm{~g})$

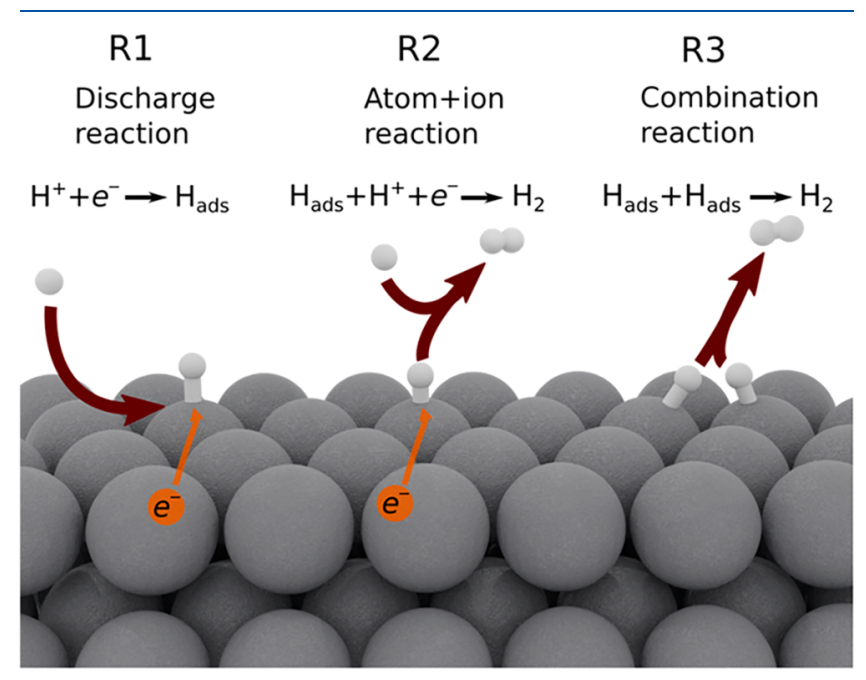

Figure 1. Three elementary reaction steps involved in the hydrogen evolution reaction. The first step is discharge reaction $\mathrm{R} 1$ proceeded by either the atom + ion reaction $\mathrm{R} 2$ or combination reaction $\mathrm{R} 3$.

Here, $\mathrm{H}^{+}$is a proton, $\mathrm{e}^{-}$is an electron from the catalyst, and $\mathrm{H}_{\text {ads }}$ is an adsorbed hydrogen atom. While both reactions R1 and R2 are electron-transfer reactions, combination reaction R3 is purely chemical and does not involve any electron transfer.

The first reaction step (R1) in HER is the adsorption of a proton followed by either an atom + ion desorption (R2) or a combination desorption ( $\mathrm{R} 3$ ) reaction. $\mathrm{R} 1$ and $\mathrm{R} 2$ involve only one adsorption site, while $\mathrm{R} 3$ is a combination reaction involving two adsorbed hydrogen atoms on neighboring sites. In the present model, we do not restrain the reaction path to either R1 $\rightarrow \mathrm{R} 2$ or R1 $\rightarrow \mathrm{R} 3$; instead, we let all elementary reaction steps contribute to the overall HER.

2.3. Hydrogen Adsorption Energies and Equilibrium Coverages. The hydrogen adsorption energy and thus $\Delta G_{\mathrm{H}}$ have been well established as descriptors for the exchange current density. ${ }^{9,28}$ According to the Sabatier principle, optimal binding energies of reactants should not be too weak (slow adsorption) or too strong (slow desorption), ${ }^{29}$ and thus if the binding energy is just about right (corresponding to a $\Delta G_{\mathrm{H}}$ of around zero), then hydrogen atoms will both easily adsorb on the metal and desorb as hydrogen gas. According to the Langmuir adsorption model, the equilibrium coverage $\left(\theta_{\text {tot }}^{*}\right)$ of a material is dependent on $\Delta G_{\mathrm{H}}$ as in eq $1 .{ }^{21,30}$ In this study, we let $\theta_{\text {tot }}^{*}$ be a fraction of one monolayer (ML). Typically, for metals, when the hydrogen coverage increases, $\Delta G_{\mathrm{H}}$ weakens (larger positive values) as a result of adsorbate interactions, ${ }^{21}$ and for heterogeneous structures, $\Delta G_{\mathrm{H}}$ also varies over the catalyst surface, making it essential to investigate all adsorption sites. All $\Delta G_{\mathrm{H}}$ values in this study are obtained using the CHE model ${ }^{16}$ as described in the Methods section 2.1.

Here we propose a two-step method that carefully finds the $\theta_{\text {tot }}^{*}$ and the corresponding $\Delta G_{\mathrm{H}}$ for heterogeneous materials. The algorithm of the method is briefly illustrated in Figure 2. In step 1 , each adsorption site $\left(i=1 \ldots \mathrm{N}_{\text {sites }}\right)$ is treated individually 

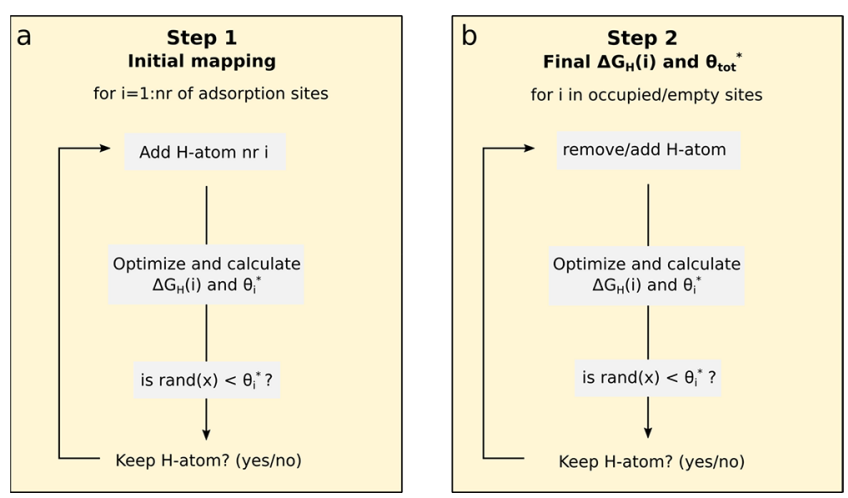

Figure 2. Algorithm to calculate the free energy of adsorption for heterogeneous structures. The algorithm is separated into two steps. Step 1: (a) All adsorption sites are initially mapped and $\Delta G_{\mathrm{H}}(i)$ is calculated. Step 2: (b) If any $\mathrm{H}$ atoms are retained from step 1 , then step 2 is needed for calculating the equilibrium values of $\Delta G_{\mathrm{H}}(i)$ and $\theta_{\text {tot }}^{*}$. The occupied sites are evaluated before the empty sites. $\operatorname{rand}(\mathrm{x})$ is a random number $[0,1]$.

using a Langmuir adsorption isotherm (eq 1), as further explained in Figure 2a. The equilibrium hydrogen occupancy, i.e., at zero overpotential with no net reaction rate, for each adsorption site $\left(\theta_{i}^{*} \in[0,1]\right)$ is thus obtained sequentially until all sites have been explored. The relationship between $\theta_{i}^{*}$ and $\Delta G_{\mathrm{H}}$ is given by eq 1

$$
\theta_{i}^{*}=\frac{p_{\mathrm{H}_{2}} \mathrm{e}^{-\Delta G_{\mathrm{H}}(i) / k T}}{1+p_{\mathrm{H}_{2}} \mathrm{e}^{-\Delta G_{\mathrm{H}}(i) / k T}}
$$

where $k$ is the Boltzmann constant and $p_{\mathrm{H}_{2}}$ and $T$ are the hydrogen pressure and temperature, set to $1 \mathrm{~atm}$ and $298.15 \mathrm{~K}$. The adsorbents are retained throughout step 1 with a probability of $\theta_{i}^{*}$ by comparing to a random number $x \in[0,1]$. A randomized exploration of the adsorption sites gives the best results by reducing clustering effects. During each adsorption, there is always a small probability that strongly adsorbing sites will be rejected and that weakly adsorbing sites will be retained. Therefore, to avoid wrong equilibrium coverages, a large simulation cell is preferable. In this study, we typically use 16 surface atoms in the simulation cell.

In step $2, \Delta G_{\mathrm{H}}(i)$ is again calculated for each site by first removing $\mathrm{H}$ atoms from all occupied sites, followed by adding $\mathrm{H}$ atoms to all empty sites from the structure saved from step 1, according to Figure $2 \mathrm{~b}$. In step 2, the probabilities of each site being occupied is again evaluated to get a more accurate $\theta_{\text {tot }}^{*}$ since step 1 often overestimates the coverage. As an example, the $\theta_{\text {tot }}^{*}$ of $1 \mathrm{ML}$ ( 16 out of $16 \mathrm{~N}_{\text {sites }}$ ) obtained in step 1 for the $\mathrm{Pt}_{111}$ surface is corrected to a final $\theta_{\text {tot }}^{*}$ of $0.81 \mathrm{ML}$ after step 2 (13 out of $\left.16 N_{\text {sites }}\right)$, in good agreement with the theoretical $\theta_{\text {tot }}^{*}(0.82$ ML) calculated with eq 1 and with experimental results. ${ }^{31-33}$

2.4. Microkinetic Model of HER for Heterogeneous Materials. The algorithm of the microkinetic model used to describe the reaction rates and evaluate the potential-dependent hydrogen coverages and current densities is displayed in Figure 3. The total hydrogen coverage of a material is obtained by averaging the individual hydrogen occupancies using eq 2 .

$$
\theta_{\text {tot }}=\frac{1}{N_{\text {sites }}} \sum_{i}^{N} \theta_{i}
$$

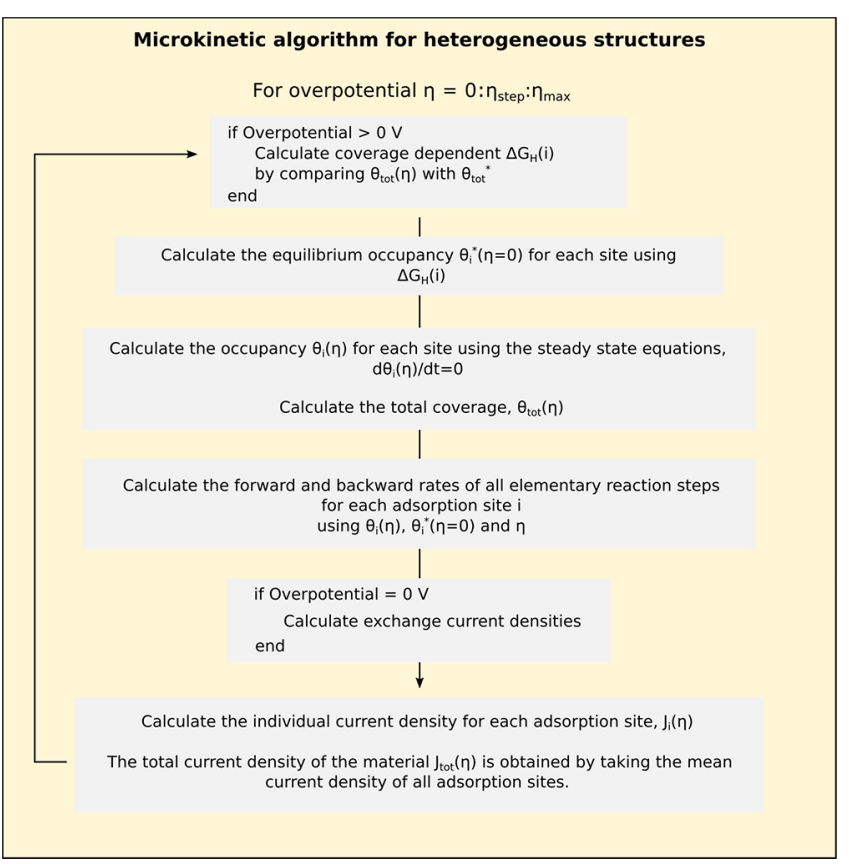

Figure 3. Algorithm used with the microkinetic model to obtain potential-dependent current densities and hydrogen coverages of heterogeneous materials.

As mentioned, $\Delta G_{\mathrm{H}}(i)$ depends on the out-of-equilibrium hydrogen coverage $\left(\theta_{\text {tot }}\right)$ of the material as a result of adsorbate interactions. Consequently, $\Delta G_{\mathrm{H}}(i)$ must be updated at each overpotential step since $\theta_{\text {tot }}$ increases with overpotential. The coverage dependency of the adsorption energy was considered by linearly modifying $\Delta G_{\mathrm{H}}$ at equilibrium coverage $\theta_{\text {tot }}^{*}$ as indicated in eq 3 , similar to Frumkin isotherms, ${ }^{34,35}$

$$
\Delta G_{\mathrm{H}}\left(i, \theta_{\text {tot }}\right)=\Delta G_{\mathrm{H}}\left(i, \theta_{\text {tot }}^{*}\right)+\left[\theta_{\text {tot }}-\theta_{\text {tot }}^{*}\right] r
$$

where $r$ is the coverage dependency between $\theta_{\text {tot }}$ and $\Delta G_{\mathrm{H}}(i)$, which is material-dependent and was found to be $0.20 \mathrm{eV} / \theta_{\text {tot }}$ for $\mathrm{Pt}_{111}$ and $0.32 \mathrm{eV} / \theta_{\text {tot }}$ for $\mathrm{Ni}_{111}$, as obtained after step 1 in section 2.3. We also calculated $r$ to be $0.25 \mathrm{eV} / \theta_{\text {tot }}$ for $\mathrm{Au}_{111}$ by evaluating $\Delta G_{\mathrm{H}}(i)$ while increasing the coverage.

Continuing with adsorption reaction step R1, the forward and backward rate equations can be described as in eqs 4 and 5, respectively.

$$
\begin{aligned}
& \overrightarrow{v_{1}}=\frac{k T}{h} a_{\mathrm{H}}\left(1-\theta_{i}\right) \mathrm{e}^{-\left(\vec{\Delta} G_{1}^{0}+\alpha \phi e_{0}\right) / k T} \\
& \overleftarrow{v_{1}}=\frac{k T}{h} \theta_{i} \mathrm{e}^{-\left(\bar{\Delta} G_{1}^{0}-(1-\alpha) \phi e_{0}\right) / k T}
\end{aligned}
$$

Here, the inner potential between the material and solution $(\phi)$ is the sum of the equilibrium potential $\left(\phi^{*}\right)$ and overpotential $(\eta): \phi=\phi^{*}+\eta \cdot \vec{\Delta} G_{1}^{0}$ and $\overleftarrow{\Delta} G_{1}^{0}$ are the standard free energies of activation for the discharge reaction when $\phi=0$. Parameter $a_{\mathrm{H}}$ is the activity of protons, and $\alpha$ is the charge-transfer coefficient, which are fixed throughout the calculations to 1 and 0.5 , respectively. Changing the transfer coefficient from 0.5 will result in "tilting" the volcano-like relation between $i_{0}$ and $\Delta G_{\mathrm{H}}$, making the left and right sides asymmetrical over $\Delta G_{\mathrm{H}}$, which will affect both the exchange current densities and Tafel slopes.

Using a derivation similar to that of Parsons, ${ }^{9}$ we evaluate the forward and backward rates under equilibrium conditions, i.e., setting $\overrightarrow{v_{1}}=\overleftarrow{v_{1}}, \theta_{i}=\theta_{i}^{*}$, and $\eta=0$. There is then no net reaction 
since the forward and backward reaction rates are equal. Setting up this criterion enables us to write $\exp \left(\phi^{*} e_{0} / k T\right)$ in terms of $\theta^{*}$ or $\left(\Delta G_{\mathrm{H}}\right)$ that can be evaluated with eq 1 but also to calculate the exchange current density $\left(i_{0}\right)$. Accordingly, after rewriting eqs 4 and 5, we obtain the rate equations for R 1 as described in eqs 6 and 7

$$
\begin{aligned}
\vec{v}_{1}(i) & =G 1 a_{\mathrm{H}}\left(1-\theta_{i}\right) a_{\mathrm{H}}{ }^{-\alpha}\left(\frac{1-\theta_{i}^{*}}{\theta_{i}^{*}}\right)^{-\alpha} \mathrm{e}^{-\alpha \eta e_{0} / k T} \\
& =\overrightarrow{k 1}\left(1-\theta_{i}\right) \\
\overleftarrow{v_{1}}(i) & =G 1 \theta_{i} a_{\mathrm{H}}{ }^{(1-\alpha)}\left(\frac{1-\theta_{i}^{*}}{\theta_{i}^{*}}\right)^{(1-\alpha)} \mathrm{e}^{(1-\alpha) \eta e_{0} / k T}=\overleftarrow{k 1} \theta_{i}
\end{aligned}
$$

where

$$
G 1=\frac{k T}{h} \mathrm{e}^{\left.-\left[(1-\alpha) \vec{\Delta} G_{1}^{0}+\alpha \bar{\Delta} G_{1}^{0}\right) / k T\right]}
$$

The potential-dependent coverages $\left(\theta_{i}\right)$ are calculated at each overpotential step, as explained later. Here we see that parameter $G 1$ is independent of $\Delta G_{\mathrm{H}}(i)$. In all rate equations, we let the equilibrium $\Delta G_{\mathrm{H}}(i)$ (and therefore $\theta_{i}^{*}$ ) change with $\theta_{\text {tot }}$ according to eq 3 along with increasing overpotential. Using a similar derivation, the rate equations $\left(\overrightarrow{v_{2}}, \overleftarrow{v_{2}}\right)$ for the atom + ion desorption step (R2) are obtained as in eqs 8 and 9.

$$
\begin{aligned}
\overrightarrow{v_{2}}(i) & =G 2 a_{\mathrm{H}} \theta_{i}\left(\frac{a_{\mathrm{H}}}{p_{\mathrm{H} 2}}\right)^{-\beta}\left(\frac{\theta_{i}^{*}}{1-\theta_{i}^{*}}\right)^{-\beta} \mathrm{e}^{-\beta \eta e_{0} / k T}=\overrightarrow{k 2} \theta_{i} \\
\overleftarrow{v_{2}}(i) & =G 2 p_{\mathrm{H} 2}\left(1-\theta_{i}\right)\left(\frac{a_{\mathrm{H}}}{p_{\mathrm{H} 2}}\right)^{(1-\beta)}\left(\frac{\theta_{i}^{*}}{1-\theta_{i}^{*}}\right)^{(1-\beta)} \mathrm{e}^{(1-\beta) \eta e_{0} / k T} \\
& =\overleftarrow{k 2}\left(1-\theta_{i}\right)
\end{aligned}
$$

where

$$
G 2=\frac{k T}{h} \mathrm{e}^{\left.-\left[(1-\beta) \vec{\Delta} G_{2}^{0}+\beta \bar{\Delta} G_{2}^{0}\right) / k T\right]}
$$

Since the combination desorption reaction step (R3) is a pure chemical step and does not depend on the overpotential, the rate equations can be written directly as presented in eqs 10 and 11 without calculating the equilibrium potential. Here we write the equations using $\Delta G_{\mathrm{H}}(i)$ instead of $\theta_{i}^{*}$.

$$
\begin{aligned}
& \overrightarrow{v_{3}}(i)=G 3 \theta_{i} \mathrm{e}^{\gamma \Delta G_{\mathrm{H}}(i) / k T} \max \left\{\theta_{\left(j, j+1, \ldots, N_{\mathrm{nb}}\right.} \mathrm{e}^{\gamma \Delta G_{\mathrm{H}}\left(j, j+1, \ldots, N_{\mathrm{nb}}\right) / k T}\right\}=\overrightarrow{k 3} \theta_{i} \\
& \overleftarrow{v_{3}}(i)=G 3 p_{\mathrm{H}_{2}}\left(1-\theta_{i}\right) \mathrm{e}^{-(1-\gamma) \Delta G_{\mathrm{H}}(i) / k T} \\
& \max \left\{\left(1-\theta_{\left(j, j+1, \ldots, N_{\mathrm{nb}}\right)}\right) \mathrm{e}^{-(1-\gamma) \Delta G_{\mathrm{H}}\left(j, j+1, \ldots, N_{\mathrm{nb}}\right) / k T}\right\} \\
& =\overleftarrow{k 3}\left(1-\theta_{i}\right)
\end{aligned}
$$

where

$$
G 3=\frac{k T}{h} \mathrm{e}^{-\Delta G_{3}^{0} / k T}
$$

In eqs 10 and 11 , we have modified the conventional way of writing the combination reaction $\left(\propto \theta^{2} \exp \left(2 \gamma \Delta G_{\mathrm{H}} / k T\right)\right)$ to include the dependency of neighboring atoms $j$ since we evaluate each rate equation for every adsorption site $i$. Since the desorption of $\mathrm{H}_{2}$ involves another adsorbate, index $j$ spans over the adjacent sites to $i$, where $N_{\mathrm{nb}}$ is the total number of adjacent sites. Site $j$ with the fastest rate is thus included in the combination reaction rate of site $i$. Here we let the rate of the combination reaction affect the coverage by a factor of 1 for each adsorption site, similar to that for other reactions. The motivation is that we study the coverages on the atomic scale for each site, where each site involves only a single hydrogen, even though the reaction releases two hydrogen atoms in total. By counting the desorption rate for all sites sequentially, we finally get the total desorption rate from the combination reaction.

Since discharge reaction $\mathrm{R} 1$ is considered to be faster than the other two elementary reaction steps, the hydrogen coverage will increase with the overpotential until the forward rates of all three reaction steps are in equilibrium. ${ }^{9,10,41}$ Therefore, the potentialdependent coverage, $\theta_{i}$, must be solved at each overpotential using the steady state condition $\mathrm{d} \theta_{i} / \mathrm{d} t=0$. To calculate this condition, we include all three reaction steps and let the HER occur simultaneously through both R1 $\rightarrow$ R2 and R1 $\rightarrow$ R3 pathways. The steady-state condition is then found by adding all adsorption and subtracting all desorption rates as in eq 12 .

$$
\frac{\mathrm{d} \theta_{i}}{\mathrm{~d} t}=0=(\overrightarrow{k 1}+\overleftarrow{k 2}+\overleftarrow{k 3})\left(1-\theta_{i}\right)-(\overleftarrow{k 1}+\overrightarrow{k 2}+\overrightarrow{k 3}) \theta_{i}
$$

By rewriting this equation, we get

$$
\theta_{i}=\frac{(\overrightarrow{k 1}+\overleftarrow{k 2}+\overleftarrow{k 3})}{(\overrightarrow{k 1}+\overleftarrow{k 1}+\overleftarrow{k 2}+\overrightarrow{k 2}+\overleftarrow{k 3}+\overrightarrow{k 3})}
$$

As evident in eqs $6-11$, both equilibrium and potentialdependent coverages are included when calculating the reaction rates. The faradaic current that each site contributes within HER is found by multiplying the net reaction rates $\left(v_{x}=\overrightarrow{v_{x}}-\overleftarrow{v_{x}}\right)$ that involve electrons by the number of electrons involved. We then get the current as $I_{x}(\mathrm{~A} /$ site $)=v_{x} z e_{0}$, where $e_{0}$ is the elementary charge and $z$ is the number of electrons involved. In all HER electron-transfer reaction steps, $z=1$. The total electrical current in units of current density $\left(\mathrm{A} / \mathrm{cm}^{2}\right)$ is then obtained by averaging $I_{x}$ over all $N_{\text {sites, }}$, which is finally multiplied by the number of adsorption sites per area $\left(\right.$ sites $\left./ \mathrm{cm}^{2}\right)$, e.g., $\sim 1.5 \times 10^{15}$ for $\mathrm{Pt}_{111}$. To account for deteriorating effects emerging from vacancies or less-dense structures, the number of adsorption sites per area should be changed when appropriate.

The exchange current density is defined as when the net current density is zero, i.e., all forward and backward reactions are equal. Therefore, the $i_{0}$ of each elementary reaction step can be obtained using the forward reaction rates at $\eta=0$, which gives the overall $i_{0}$ of the material using rate-determining reactions $\mathrm{R} 2$ and $\mathrm{R} 3$ according to eq 13

$$
i_{0}=\left(2 \overrightarrow{v_{2}}+\overrightarrow{v_{3}}\right) e_{0}
$$

where the term $\overrightarrow{v_{2}}+\overrightarrow{v_{3}}$ corresponds to the adsorption rate $\overrightarrow{v_{1}}$ and the additional $\overrightarrow{v_{2}}$ is the desorption rate of $\mathrm{R} 2$. The $i_{0}$ given in eq 13 corresponds to the experimentally obtained $i_{0}$ for the full HER.

\section{RESULTS}

3.1. Equilibrium Hydrogen Coverage. We now turn to the presentation of more detailed results of the equilibrium coverages and $\Delta G_{\mathrm{H}}(i)$ for the selected adsorption sites in periodic slabs of $\mathrm{Pt}_{111}, \mathrm{Ni}_{111}$, and $\mathrm{Au}_{111}$, each containing $16 \times 4$ 

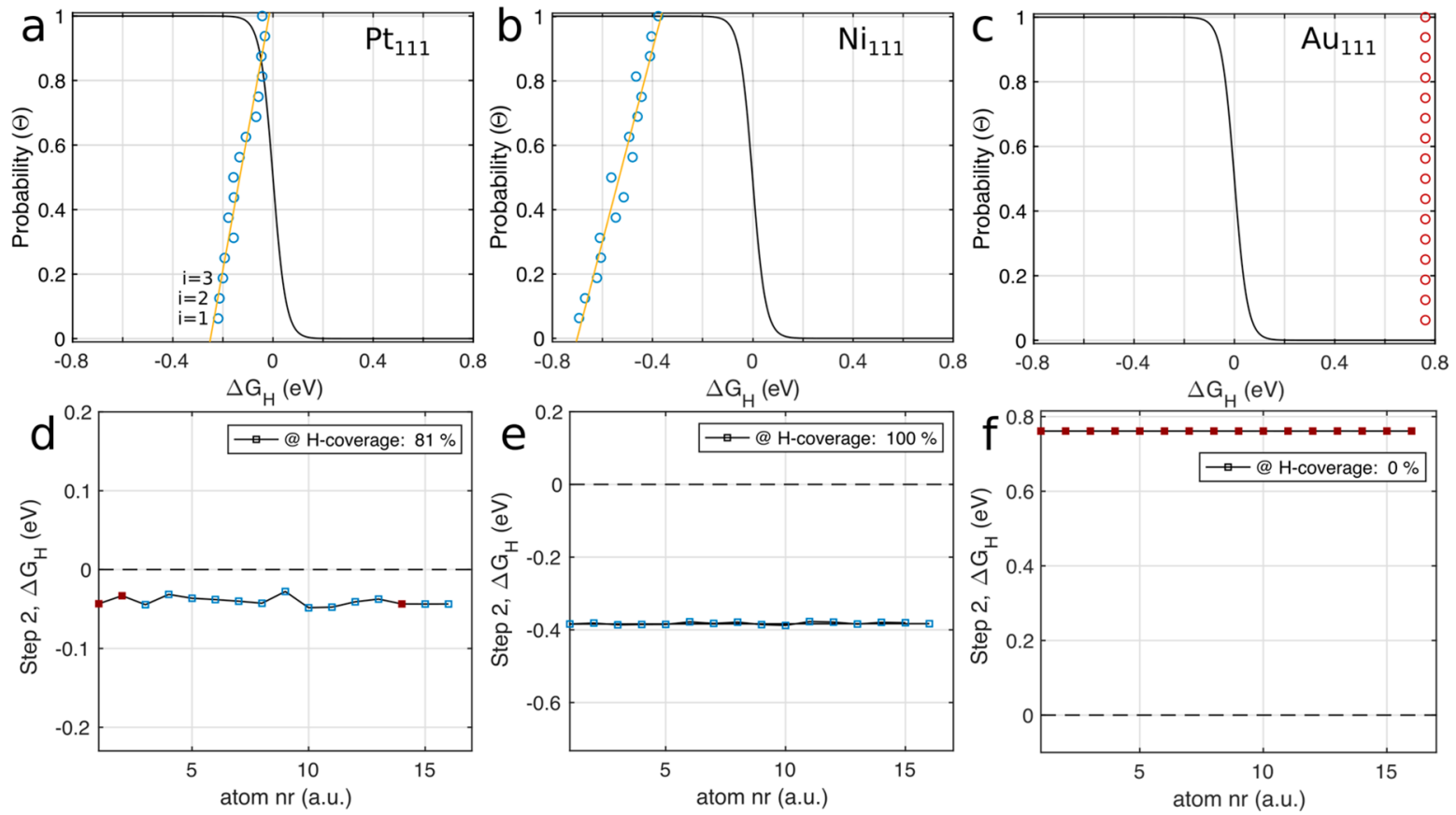

Figure 4. Data from $\mathrm{Pt}_{111}, \mathrm{Ni}_{111}$, and $\mathrm{Au}_{111}$ surfaces, each with four atomic layers. (a-c) Step 1: the blue circles mark where the $\mathrm{H}$ atoms were kept, and red circles mark where $\mathrm{H}$ atoms were not kept. Therefore, for each blue circle the total coverage is increasing. The data in panels a and $\mathrm{b}$ are also fitted with linear equations as shown, with slopes of 0.20 and $0.32 \mathrm{eV} / \theta^{*}$ for $\mathrm{Pt}_{111}$ and $\mathrm{Ni}_{111}$ respectively. (d-f) Step 2: the final $\Delta G_{\mathrm{H}}$ used for the microkinetic modeling. The equilibrium coverages obtained for each metal after step 2 are also displayed. The red squares mark where $\mathrm{H}$ atoms were removed, and the blue squares mark when $\mathrm{H}$ atoms were kept.

atoms. In Figure $4(\mathrm{a}-\mathrm{c})$, the data from step 1 is plotted for each adsorption site, along with $\theta_{i}^{*}$ from eq 1 (black line) as a function of $\Delta G_{\mathrm{H}}(i)$. For sites with strong adsorption energies (negative $\Delta G_{\mathrm{H}}$ ), the probability of these being covered is close to 1 , while for weak adsorption energies (positive $\Delta G_{\mathrm{H}}$ ) the probability approaches 0 very quickly. It is generally only in a narrow range of $\Delta G_{\mathrm{H}}$ values of $\pm 0.1 \mathrm{eV}$ where the probability significantly deviates from 0 or 1 and where $\theta_{\text {tot }}^{*}$ is different from 0 or $1 \mathrm{ML}$. This is one of the reasons why there are relatively few good catalysts despite the large diversity of materials. In Figure $4(\mathrm{~d}-\mathrm{f})$, we calculated the final $\Delta G_{\mathrm{H}}$ and the $\theta_{\text {tot }}^{*}$ using step 2 for the metals. It is notable that even for a homogeneous surface such as $\mathrm{Pt}_{111}, \Delta G_{\mathrm{H}}(i)$ varies along the surface when its partially covered with hydrogen atoms as indicated in Figure 4d, showing that a single value for $\Delta G_{\mathrm{H}}$ is insufficient to describe the total activity. Similarly, we emphasize that evaluating all sites for heterogeneous structures also is essential since for these structures larger variations are usually found. In Table 1, detailed data from similar calculations for several metals are presented. We note that for metals with very weak adsorption $\left(\Delta G_{\mathrm{H}} \gg 0\right)$ for the on-top sites, such as copper and gold, we choose those sites when determining the activity, even though other sites have more energetically favorable $\Delta G_{\mathrm{H}}$ values. We have noticed that for such metals the on-top sites represent the experimental activity better (Table 1 ). It is possible that the $\mathrm{H}$ atoms desorb from on-top sites during the adsorption process before diffusing through any of the pathways OT $\rightarrow \mathrm{fcc}, \mathrm{OT} \rightarrow$ hcp or OT $\rightarrow$ bridge, in line with the low hydrogen coverage for such metals. In the table, experimental current densities $i_{0}$ are also presented that were taken from high-purity polycrystalline metals with measurements made under rigorous conditions that were carefully revised in a comparative study by Trasatti. ${ }^{36}$ However, regarding platinum, more recent studies using microelectrodes have attained $i_{0}$ values that are up to 25 times higher, proving the large variations in $i_{0}$ for $\mathrm{Pt}$ in the literature. $^{37,38}$ For $\mathrm{Pt}$, we have therefore taken $i_{0}$ from a more representative study made by Parsons that was measured under high-purity conditions via methods similar to those for the other metals. $^{39}$

3.2. Volcano Plots. When $i_{0}$ is plotted against $\Delta G_{\mathrm{H}}$, a volcano-shaped relation is observed, centered at $\Delta G_{\mathrm{H}}=0 \mathrm{eV}$. In this section, we present how the volcano plot is fitted against experimental values of exchange current densities and Tafel slopes. The only parameters for the rate equations (eq 6-11) that are fitted to experimental data are $G 1, G 2$, and $G 3$, which adjust the magnitude of the exchange current density of each elementary reaction step. A common agreement is that the discharge reaction is fast and that the hydrogen coverage therefore increases with overpotential. ${ }^{9,10,41}$ Since $G 1$ controls the hydrogen discharge reaction, it is then the relative magnitude of $G 1$ that determines the potential range in which the coverage increases, and a larger magnitude of $G 1$ relative to $G 2$ or $G 3$ indicates the extent of such an increase.

Since we showed that $G 1$ is the largest, it is then $G 2$ and $G 3$ that determine the value of the total exchange current and therefore the magnitude of the total HER. As a consequence of the potential independency for R3, if we set $G 3 \gg G 2$, then materials with slightly negative $\Delta G_{\mathrm{H}}$ will obtain a current density that initially stalls at low $\eta$ and is able to increase only when R2 becomes faster than R3 at higher $\eta$. This effect causes a saddlelike polarization curve and is not seen experimentally that we are aware of. Therefore, we set the value of $G 3$ only slightly higher than G2, which allows for Tafel slopes of $30 \mathrm{mV} / \mathrm{dec}$ for materials near the top of the volcano while avoiding saddlelike polarization curves. The absolute values of $G 2$ and $G 3$ are thereafter fitted to represent the experimental $i_{0}$ of Pt. The absolute value of $G 1$ is finally fitted according to experimental data, from where two Tafel lines have been observed at different 
Table 1. Theoretically Calculated Lattice Constants, Hydrogen Adsorption Free Energies $\left(\Delta G_{\mathrm{H}}\right)$, Hydrogen Coverage, and Exchange Current Densities $\left(i_{0}\right)$ for Different Metals, Together with Experimental Values of $i_{0}{ }^{a}$

\begin{tabular}{|c|c|c|c|c|c|c|c|c|c|c|c|c|}
\hline \multirow{2}{*}{$\begin{array}{l}\text { metal } \\
\text { Pt111 }\end{array}$} & \multirow[t]{2}{*}{$a(\AA)$} & \multirow{2}{*}{$\begin{array}{c}\text { 3-fold fcc } \\
(\mathrm{eV})^{b} \\
-\mathbf{0 . 2 2}\end{array}$} & \multirow{2}{*}{$\begin{array}{l}\text { 3-fold hcp } \\
(\mathrm{eV})^{b} \\
-0.15\end{array}$} & \multirow{2}{*}{$\begin{array}{l}\mathrm{OT}^{b} \\
(\mathrm{eV})^{b} \\
-0.19\end{array}$} & \multirow{2}{*}{$\begin{array}{l}\text { bridge } \\
(\mathrm{eV})^{b} \\
-0.20^{i}\end{array}$} & \multirow[t]{2}{*}{$\begin{array}{l}\text { 4-fold } \\
(\mathrm{eV})^{b}\end{array}$} & \multirow{2}{*}{$\begin{array}{c}\theta_{\text {tot }}^{* c} / \theta_{\text {totDFT }}^{*} \\
(\mathrm{ML}) \\
0.82 / 0.81\end{array}$} & \multicolumn{2}{|c|}{$\begin{array}{c}\text { projected } \\
\Delta G_{\mathrm{H}}(\mathrm{eV})^{d}\end{array}$} & \multicolumn{2}{|c|}{$\underset{\substack{\text { mean } \\
i_{0}-\left(\log _{10}(A / c-\right.}}{\left.\left.\mathrm{m}^{2}\right)\right)}$} & \multirow{2}{*}{$\begin{array}{c}\exp i_{0}-\left(\log _{10}\right. \\
\left.\left(A / \mathrm{cm}^{2}\right)\right) \\
2.60^{39,40}\end{array}$} \\
\hline & & & & & & & & -0.04 & -0.07 & 2.38 & 2.68 & \\
\hline Pt100 & 3.99 & & & -0.17 & -0.34 & move $^{i}$ & $1.00 / 1.00$ & -0.26 & & 4.35 & & \\
\hline Nil11 & & -0.69 & -0.69 & -0.19 & -0.69 & & $1.00 / 1.00$ & -0.38 & -0.41 & 5.43 & 5.66 & $5.25^{36}$ \\
\hline Nil00 & 3.53 & & & -0.17 & -0.57 & -0.77 & $1.00 / 1.00$ & -0.47 & & 6.17 & & \\
\hline Au111 & & +0.67 & +0.68 & +0.76 & +0.67 & & $0.00 / 0.00$ & +0.76 & +0.50 & 8.63 & 6.42 & $6.50^{36}$ \\
\hline Au100 & 4.18 & & & +0.46 & +0.23 & +0.40 & $0.00 / 0.00$ & +0.46 & & 6.12 & & \\
\hline Ag111 & & +0.38 & +0.40 & +0.92 & +0.49 & & $0.00 / 0.00$ & +0.92 & +0.79 & 9.95 & 8.89 & $7.90^{36}$ \\
\hline Ag100 & 4.15 & & & +0.76 & +0.34 & +0.29 & $0.00 / 0.00$ & +0.76 & & 8.61 & & \\
\hline Cu111 & & +0.02 & +0.09 & +0.61 & +0.01 & & $0.00 / 0.00$ & +0.61 & +0.56 & 7.38 & 6.91 & $7.8^{36}$ \\
\hline Cu100 & 3.71 & & & +0.53 & +0.14 & +0.05 & $0.00 / 0.00$ & +0.53 & & 6.68 & & \\
\hline Col11 & & -0.61 & -0.68 & move $^{i}$ & move $^{i}$ & & $1.00 / 1.00$ & -0.36 & -0.39 & 5.24 & 5.44 & $5.30^{36}$ \\
\hline $\mathrm{Co}_{0001^{e}}$ & 2.53 & -0.53 & -0.62 & -0.06 & $>$ move & & $1.00 / 1.00$ & -0.43 & & 5.83 & & \\
\hline $\operatorname{Ir} 111$ & & -0.06 & -0.03 & -0.16 & -0.06 & & $0.78 / 0.75$ & -0.05 & -0.08 & 2.47 & 2.77 & $3.60^{36}$ \\
\hline $\operatorname{Ir} 100$ & 3.89 & & & -0.32 & -0.46 & move $^{i}$ & $1.00 / 1.00$ & -0.41 & & 5.67 & & \\
\hline Pd111 & & -0.37 & -0.33 & +0.20 & move $^{i}$ & & $1.00 / 1.00$ & -0.26 & -0.25 & 4.38 & 4.26 & $3.10^{36}$ \\
\hline Pd100 & 4.01 & & & +0.31 & move $^{i}$ & -0.26 & $1.00 / 1.00$ & -0.24 & & 4.17 & & \\
\hline Rh111 & & -0.25 & -0.24 & +0.14 & -0.17 & & $1.00 / 1.00$ & -0.16 & -0.20 & 3.56 & 3.85 & $3.50^{36}$ \\
\hline Rh100 & 3.86 & & & +0.09 & move $^{i}$ & -0.31 & $1.00 / 1.00$ & -0.36 & & 5.24 & & \\
\hline Al111 & & +0.66 & move $^{i}$ & +0.52 & +0.63 & & $0.00 / 0.00$ & +0.52 & +0.55 & 6.59 & 6.82 & $8.00^{36}$ \\
\hline Al100 & 4.09 & & & +0.61 & +0.38 & move $^{i}$ & $0.00 / 0.00$ & +0.61 & & 7.35 & & \\
\hline Mo110 & & & & +0.31 & -0.35 & $-0.41^{g}$ & $1.00 / 1.00$ & -0.35 & -0.23 & 5.15 & 4.11 & $7.30^{36}$ \\
\hline Mo100f & 3.18 & & & move $^{i}$ & -0.88 & move $^{i}$ & $1.00 / 1.00$ & -0.19 & & 3.83 & & \\
\hline $\mathrm{Nb} 110^{f}$ & & & & +0.45 & -0.37 & $-0.58^{g}$ & $1.00 / 1.00$ & -0.48 & -0.51 & 6.25 & 6.47 & $8.40^{36}$ \\
\hline $\mathrm{Nb} 100^{f}$ & 3.34 & & & move $^{i}$ & -1.25 & -1.30 & $1.00 / 1.00$ & -0.56 & & 6.94 & & \\
\hline $\operatorname{Re} 111^{h}$ & & & & & & & $1.00 / 1.00$ & -0.18 & -0.18 & 3.69 & 3.69 & $3.00^{36}$ \\
\hline Re100 & & & & & & & & & & & & \\
\hline $\mathrm{W} 110^{h, e}$ & & & & & & & $1.00 / 1.00$ & -0.56 & -0.56 & 6.93 & 6.93 & $6.40^{36}$ \\
\hline
\end{tabular}

${ }^{a}$ All results are from face-centered cubic (fcc) systems if not mentioned otherwise. The mean $i_{0}$ is taken from a 1:1 mixture between the tested surfaces in each metal. The surfaces are evaluated separately and combined with no interaction. The values in bold font were selected for the analysis. ${ }^{b}$ The adsorption free energy, $\Delta G_{\mathrm{H}}$, presented is the value at minimal coverage, i.e., the first adsorbed $\mathrm{H}$ atom. ${ }^{c} \theta_{\text {tot }}^{*}$ is calculated using the Langmuir adsorption isotherm with $\Delta G_{\mathrm{H}}$ from step $2 . \theta_{\mathrm{totDFT}}^{*}$ is the equilibrium coverage found at step 2 from which $\Delta G_{\mathrm{H}}$ is obtained. ${ }^{d}$ The values are taken from the mean $i_{0}$ values, which are projected onto the corresponding $\Delta G_{\mathrm{H}}$ value in the volcano plot. ${ }^{e}$ Hexagonal close-packed (hcp) system. ${ }^{f_{\mathrm{B}}}$ Body-centered cubic (bcc) system. ${ }^{g}$ These adsorption sites are 3 -fold hollow sites. ${ }^{h}$ Data are taken from ref $16 .{ }^{i}$ The $\mathrm{H}$ atoms moved to the most stable adsorption sites during optimization.
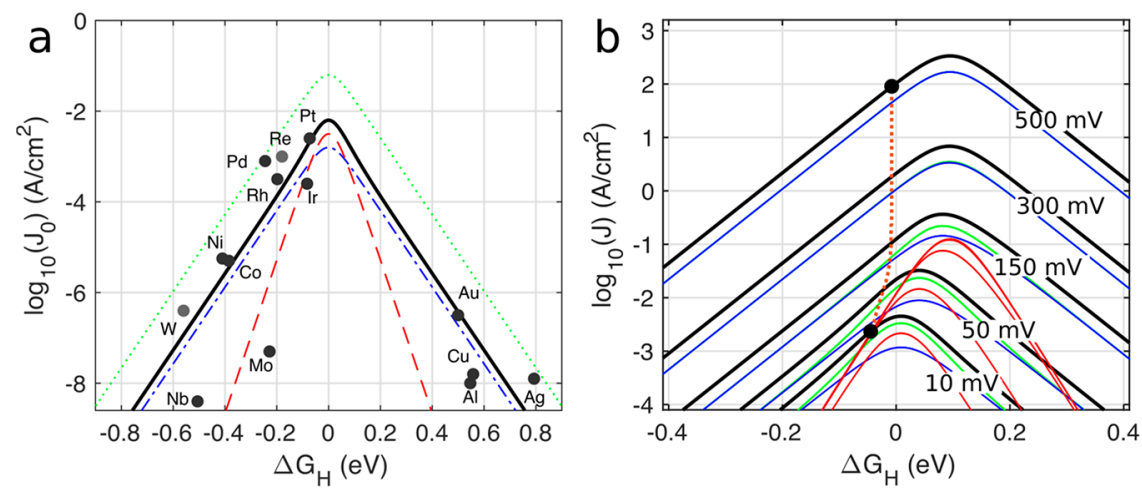

Figure 5. (a) Volcano plot of exchange currents for polycrystalline metals, showing all three reactions in HER and the total exchange current as the discharge reaction (green dotted line), the atom + ion desorption (blue dashed-dotted line), the combination reaction (red dashed line), and the total reaction (black solid line). The $\Delta G_{\mathrm{H}}$ for $\mathrm{W}$ and Re are taken from ref 16. All other $\Delta G_{\mathrm{H}}$ values are from this work. The $\Delta G_{\mathrm{H}}$ values are projected from the mean calculated exchange currents combining different surfaces. (b) Current density of each reaction plotted versus $\Delta G_{\mathrm{H}}$ at different overpotentials. The dotted orange line with filled black circles as end points marks $\mathrm{Pt}_{111}$ in each overpotential step.

current density ranges for the same metal. The value was chosen from the difference between the total $i_{0}$ and $i_{0}$ of $\mathrm{R} 1$ according to
Figure S3 and was found to be 17.8 times higher for R1. In conclusion, the parameters are fitted as 

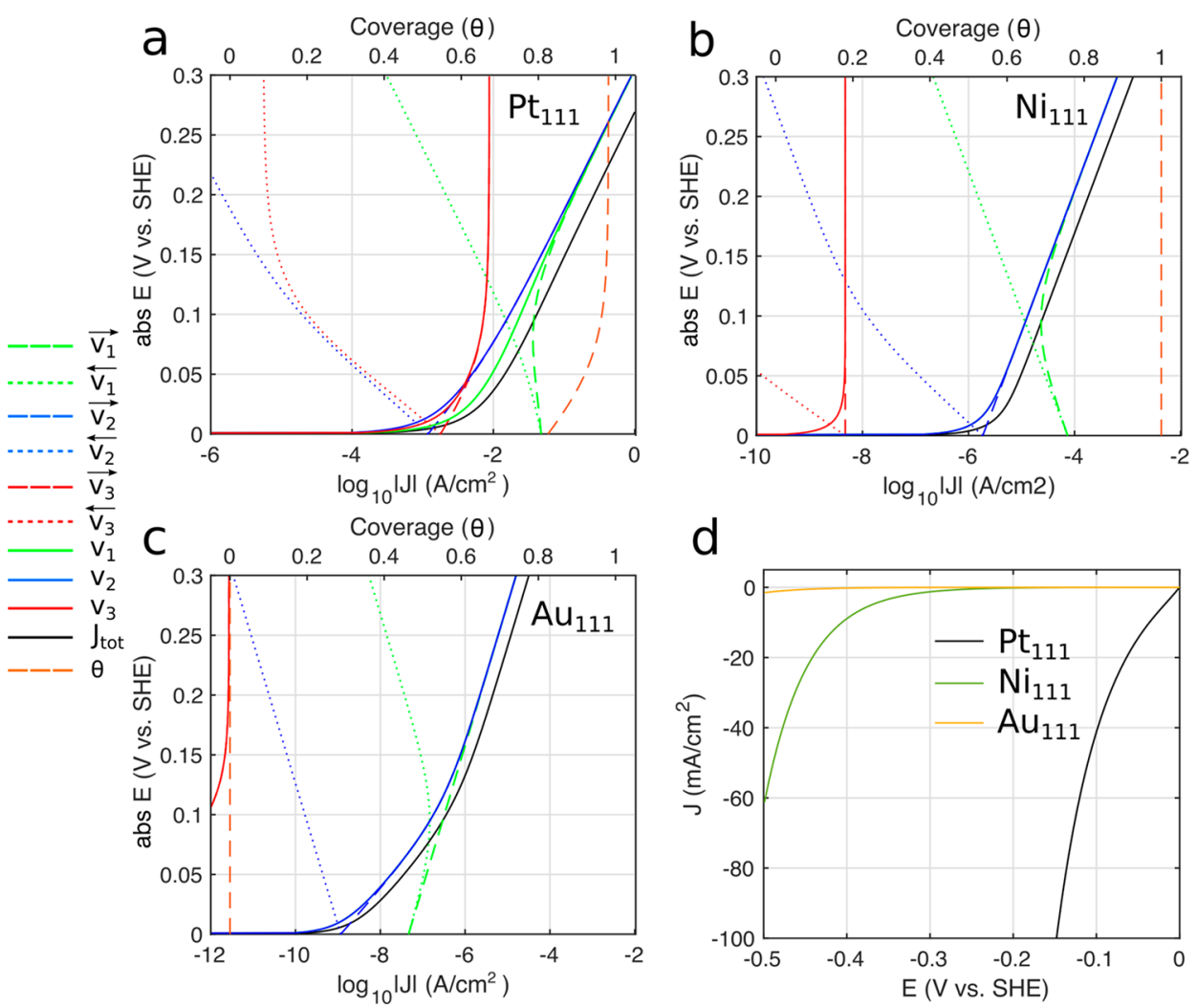

d

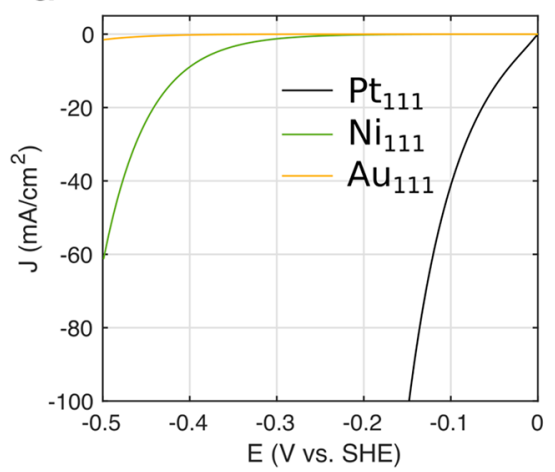

Figure 6. $(\mathrm{a}-\mathrm{c})$ Detailed Tafel plots showing Tafel lines $\left(v_{x}\right)$ corresponding to each reaction (forward, backward, and net directions) are shown together with the total Tafel line $\left(J_{\text {tot }}\right)$ and the hydrogen coverages for $\mathrm{Pt}_{111}, \mathrm{Ni}_{111}$, and $\mathrm{Au}_{111}$ respectively. (d) Polarization curves for all metals.

$$
\begin{aligned}
& G 1=10^{-0.9} /\left(1.5 \times 10^{15}\right) \\
& G 2=10^{-2.5} /\left(1.5 \times 10^{15}\right) \\
& G 3=10^{-1.9} /\left(1.5 \times 10^{15}\right)
\end{aligned}
$$

where the denominator $\left(1.5 \times 10^{15}\right)$ is the number of adsorption sites $/ \mathrm{cm}^{2}$ in $\mathrm{Pt}_{111}$ and is used for fitting the volcano plot.

Figure 5a shows the volcano plot where the individual $i_{0}$ for R1 (green), R2 (blue), and R3 (red) and the total $i_{0}$ (black) are plotted together with experimental data from Table 1. The agreement between these are generally very good. However, for some metals in the left branch, i.e., $\mathrm{Mo}, \mathrm{Nb}$, and $\mathrm{W}$, we note that metal oxides are usually formed in acidic solution. This complicates the analysis and could explain the discrepancy for these metals. $^{42-44}$ For example, large time variations of overpotential and anomalous Tafel lines were observed for Mo even after pre-electrolysis for $37 \mathrm{~h}$ at $7.5 \mathrm{~mA} / \mathrm{cm}^{2}$, unlike for the other metals $(\mathrm{Au}, \mathrm{Pd}, \mathrm{Rh}, \mathrm{Cu}$, and $\mathrm{Pt})$ in the study. ${ }^{45}$ Therefore, the theoretical values for metallic $\mathrm{Mo}$ and $\mathrm{Nb}$ are likely not comparable to experimental values, and possible correlation is likely fortunate for these specific metals. When the exchange currents of the metals in Figure 5a are compared to similar models commonly used in the literature, which often consider the Tafel step to be rate-determining or do not account for the charge-transfer coefficient, this model in particular gives better agreement with experimental results for materials with very high or low values of $\Delta G_{\mathrm{H}} \cdot{ }^{15,16}$ The current model can further be used at nonzero overpotentials using eq 12, where each elementary reaction step is no longer in self-equilibrium and the rate-determining steps are able to change. In Figure $5 b$, we plot the net current densities of reaction steps R1-R3 as well as the total current density with increasing $\eta$. The orange dotted line in the figure marks the activity of $\mathrm{Pt}_{111}$ at each $\eta$. As apparent in the figure, catalysts with slightly positive $\Delta G_{\mathrm{H}}$ values are most active at higher overpotentials while catalysts with $\Delta G_{\mathrm{H}}=0 \mathrm{eV}$ are ideal only at $\eta=0$.

3.3. Polarization Curves. The Tafel slopes of reactions R1, $\mathrm{R} 2$, and R3 are calculated at low $\eta$ for $\Delta G_{\mathrm{H}} \gg 0$ (low Hcoverage) to 118,40 , and $30 \mathrm{mV} / \mathrm{dec}$, respectively, consistent with previous theoretical values. ${ }^{9,10,14}$ Thereafter, at higher $\eta$, when the coverage becomes constant and the reaction is limited by R1, the Tafel slope of $\mathrm{R} 2$ increase to $118 \mathrm{mV} / \mathrm{dec}$, independently of $\Delta G_{\mathrm{H}}$. Since R3 can only increase with coverage, the Tafel slope for $\mathrm{R} 3$ is infinite at constant coverage. If we instead consider negative $\Delta G_{\mathrm{H}}$ values (high $\mathrm{H}$ coverage), then the $\Delta G_{\mathrm{H}}$ values move further away from the top of the volcano when $\eta$ increases (Figure $5 \mathrm{~b}$ ), and since the coverage quickly reaches 1 here, the Tafel slopes of R2 and R3 are 118 $\mathrm{mV} / \mathrm{dec}$ and infinite, respectively, even at low $\eta$. Examples of Tafel plots for different $\Delta G_{\mathrm{H}}$ values are shown in Figure $S 1$. For heterogeneous materials with varying $\Delta G_{\mathrm{H}}(i)$, the obtained Tafel slopes can be combinations of the previously mentioned values.

In Figure $6(\mathrm{a}-\mathrm{d})$, we present detailed Tafel plots and polarization curves for $\mathrm{Pt}, \mathrm{Ni}$, and $\mathrm{Au}$ single-metal slabs. The Tafel lines corresponding to each reaction step (backward, forward, and net reactions) are plotted as $v_{i}$, where $i$ indicates the elementary reaction number $R_{i}$. The calculated total current density is plotted as $J_{\text {tot }}$. The total hydrogen coverage, $\theta_{\text {tot }}$, for the metals is also shown in each figure. For Pt, the $\theta_{\text {tot }}$ value increases from $0.82 \mathrm{ML}$ at equilibrium to just below $1 \mathrm{ML}$ at $\eta=0.2 \mathrm{~V}$ vs $\mathrm{SHE}$ and is constant at higher overpotentials. For $\mathrm{Ni}$ and $\mathrm{Au}$, the coverage is very close to 1 and $0 \mathrm{ML}$, respectively, and the change with overpotential is almost unnoticeable on this scale. The results for $\mathrm{Pt}_{111}$ are very sensitive to small changes, and 
calculating the exchange current density from Figure $6 \mathrm{a}$ in a manner similar to what one would experimentally can give various values of $i_{0}$ due to an obvious absence of a linear region, highlighting the difficulty of measuring experimental values of $i_{0}$.

Our results for $\mathrm{Pt}_{111}$ do not display the expected linear Tafel slope of $30 \mathrm{mV} / \mathrm{dec}$, which is often found experimentally for Pt. The reason for this is that $\Delta G_{\mathrm{H}}$ for $\mathrm{Pt}_{111}$ is slightly negative, and as mentioned earlier, a Tafel slope of $30 \mathrm{mV} / \mathrm{dec}$ can be obtained only at slightly positive $\Delta G_{\mathrm{H}}$, near the top of the volcano for R3. It is possible that for $\mathrm{Pt}_{111}$ when $\theta_{\text {tot }}>\theta_{\mathrm{tot}}^{*}$, some $\mathrm{H}$ atoms adsorb on the less-favorable on-top sites and facilitate the combination reaction. ${ }^{15,41,46}$ We have therefore calculated $\Delta G_{\mathrm{H}}$ at hydrogen coverages above $\theta_{\text {tot }}^{*}$ for several adsorption sites on $\mathrm{Pt}_{111}$, as indicated by the red marks in Figure S2, which also show the final configurations and the calculated $\Delta G_{\mathrm{H}}$, along with similar calculations for other metals in Table S2. At coverages just above $\theta_{\text {tot }}^{*}$ for $\mathrm{Pt}_{111}$, the available on-top sites displayed in Figure $S 2$ have a slightly positive $\Delta G_{\mathrm{H}}$ of $0.03 \mathrm{eV}$, only $0.06 \mathrm{eV}$ more positive than the fcc sites at the same coverage. However, at coverages above $1 \mathrm{ML}$, similar as when all neighboring fcc sites are occupied, the adsorbed $\mathrm{H}$ atoms display a very weak $\Delta G_{\mathrm{H}}>0.35 \mathrm{eV}$ for on-top sites, outside the active range of $\mathrm{R} 3$. Several experimental studies have also suggested the presence of subsurface $\mathrm{H}$ atoms in $\mathrm{Pt}$ that could influence the activity. ${ }^{47-49}$ According to our results, any subsurface $\mathrm{H}$ atom in $\mathrm{Pt}_{111}$ strengthened the $\Delta G_{\mathrm{H}}$ of fcc sites by roughly $0.1 \mathrm{eV}$ (making it more negative). Conclusively, according to our calculations neither subsurface $\mathrm{H}$ atoms nor adsorbed $\mathrm{H}$ atoms above $\theta_{\text {tot }}^{*}$ could explain an experimental Tafel slope of $30 \mathrm{mV} /$ $\mathrm{dec}$ for Pt. Another alternative is that metal-solution interfaces could alter the binding energies, as suggested in experimental studies. $^{28,32}$ Even though including water molecules is computationally demanding and has previously shown only small effects in theoretical studies, ${ }^{8,15,17,18,50}$ it was recently established in an experimental study that the hydrogen binding energies increase $\left(\Delta G_{\mathrm{H}}\right.$ more negative) along with $\mathrm{pH}$ in a likely metalindependent manner. ${ }^{28}$ Therefore, it is still possible that $\Delta G_{\mathrm{H}}$ is affected by $\mathrm{pH}$-dependent water adsorption/desorption reactions. Further efforts are needed to evaluate this.

Regarding $\mathrm{Au}$, a previous experimental study found two distinct Tafel slopes at low and high current densities with values of 50 and $105 \mathrm{mV} / \mathrm{dec}$, in good agreement with our results (40 and $118 \mathrm{mV} / \mathrm{dec}$ ) and with the adsorption of $\mathrm{H}$ atoms as ratedetermining. ${ }^{51}$ Similar to other studies, Au also displays two Tafel slopes at around 70 and $100 \mathrm{mV} / \mathrm{dec}$ at low and high current denities. $^{40,45,52}$ Considering Ni, only one Tafel slope is normally observed with a value of $118 \mathrm{mV} / \mathrm{dec}$, also matching our results, with the atom + ion rate-determining desorption step. $^{40,52-55}$

3.4. Heterogeneous Molybdenum Disulfide Catalyst. Finally, we demonstrate an example of how a heterogeneous structure can be studied with the model. We tested a molybdenum disulfide $\left(\mathrm{MoS}_{2}\right)$ structure that included both the basal plane and edge atoms in the calculation. The free energies included are shown in Table 2, and the values are taken from a recent theoretical study by Kronberg et al. We selected the most energetically stable edge configurations and choose the equilibrium hydrogen coverage values of $\Delta G_{\mathrm{H}}$ for these in agreement with our model. ${ }^{56}$ We have tested three different structures: one with only basal plane atoms, one with only edge atoms, and one comprising a mixture between basal plane and edges. In the mixture, we investigate a structure with $1 \%$ edges and $99 \%$ basal plane atoms that resembles a square surface with
Table 2. Type of Adsorption Site, $\Delta G_{H}$ Value, and the Relative Amount of Each Site ${ }^{a}$

$\begin{array}{lcc}\text { adsorption site } & \Delta G_{\mathrm{H}}(\mathrm{eV}) & \text { relative amount } \\ \text { Basal Plane } & & \\ 2 \mathrm{H} \mathrm{MoS} & 1.8 & 1 \\ \text { Mo Edge } & & \\ 50 \% \text { S coverage } & 0.1 & 2 / 3 \\ 100 \% \text { S coverage } & 0.3 & 1 / 3 \\ \text { S Edge } & & 1 \\ 100 \% \text { S coverage } & -0.2 & \end{array}$

${ }^{a}$ For Mo edges, two separate types are considered with different $S$ coverage values of edge termination (50 and 100\%). The ratio between Mo and S edges is 1:1. All data are taken from refs 56 and 59 .

roughly 400 atoms $\times 400$ atoms $(130 \mathrm{~nm}$ width) where all four sides consist of edge atoms. Half of the edges are chosen as Mo edges, and the other half are chosen as $\mathrm{S}$ edges. The exact types and their relative amounts are shown in Table 2. In $2 \mathrm{H}-\mathrm{MoS}_{2}$, essentially all catalytic activity is attributed to the edges since the basal plane has very weak hydrogen adsorption and contributes only by lowering the relative density of active sites (edges) in the material. ${ }^{57,58}$ This is a consequence of the logarithmic scale in the volcano plot against $\Delta G_{\mathrm{H}}$, where a $\Delta G_{\mathrm{H}}$ difference of $0.1 \mathrm{eV}$ alters the exchange current density by roughly 1 order of magnitude, which is equivalent to reducing the density of active sites by 10 -fold. ${ }^{59}$

In Figure 7a, we show the theoretical polarization curves for $\mathrm{MoS}_{2}$, and in Table S3, we show the calculated exchange current densities and the $\eta$ needed for $10 \mathrm{~mA} / \mathrm{cm}^{2}$ for several different $\mathrm{MoS}_{2}$ configurations with different relative numbers of edge atoms. The structure with $1 \%$ edges agrees well with the activity of experimental $\mathrm{MoS}_{2}$ catalysts with $\eta=0.26 \mathrm{~V}$ at $10 \mathrm{~mA} / \mathrm{cm}^{2}$, where experimental values normally need an $\eta$ value of between 0.10 and $0.25 \mathrm{~V}^{60}$ The two circles in the figure mark where 10 $\mathrm{mA} / \mathrm{cm}^{2}$ is achieved for structures with 10 and $50 \%$ edges, corresponding to $\eta=-0.15$ and $-0.09 \mathrm{~V}$, respectively. Experimental current densities of $10 \mathrm{~mA} / \mathrm{cm}^{2}$ for the most active $\mathrm{MoS}_{2}$-based catalysts are often found in the range of $\eta=$ $0.11-0.20 \mathrm{~V}$, which agrees very well with our results, and would then have between 3 and $25 \%$ of the exposed atoms as edges if all surface atoms are inactive. ${ }^{5,58,60,61}$ One should, however, keep in mind that the electrochemical area in $\mathrm{MoS}_{2}$ catalysts is often larger than the geometrical area because of their layered morphology and that surface defects or different crystal structures could also activate the basal plane. ${ }^{59}$ The structure with a $100 \%$ basal plane performs poorly $(\eta=1.64 \mathrm{~V}$ at $10 \mathrm{~mA} /$ $\mathrm{cm}^{2}$ ), but since there are always some defects, strains, edges, or other imperfections present in real structures, such a nonideal $\mathrm{MoS}_{2}$ structure is difficult to find in the literature. However, a recent study measured edge-oxidized $2 \mathrm{H}-\mathrm{MoS}_{2}$ and reported a Tafel slope of $186 \mathrm{mV} / \mathrm{dec}$ and $\eta=0.55 \mathrm{~V}$ to reach $10 \mathrm{~mA} /$ $\mathrm{cm}^{2}{ }^{62}$ From our theoretical results, this overpotential would correspond to a structure with $0.005 \%$ edges compared to basal plane atoms using the composition in Table 2. Tafel slopes higher than theoretical values could, for example, be explained by nonconductive substrates, varying electrochemical area, diffusion limitations, or competing reactions. In Figure $7 \mathrm{~b}$, the theoretical Tafel plots of $\mathrm{MoS}_{2}$ are plotted, and the first Tafel line at low $\eta$ for the $1 \%$ edge structure agrees well with experimental Tafel slopes of $\mathrm{MoS}_{2}$ that normally lie between 40 and $70 \mathrm{mV} / \mathrm{dec}^{58,60,62,63}$ However, linear regions in the Tafel plot for the $100 \%$ edge system are difficult to find for the low- 

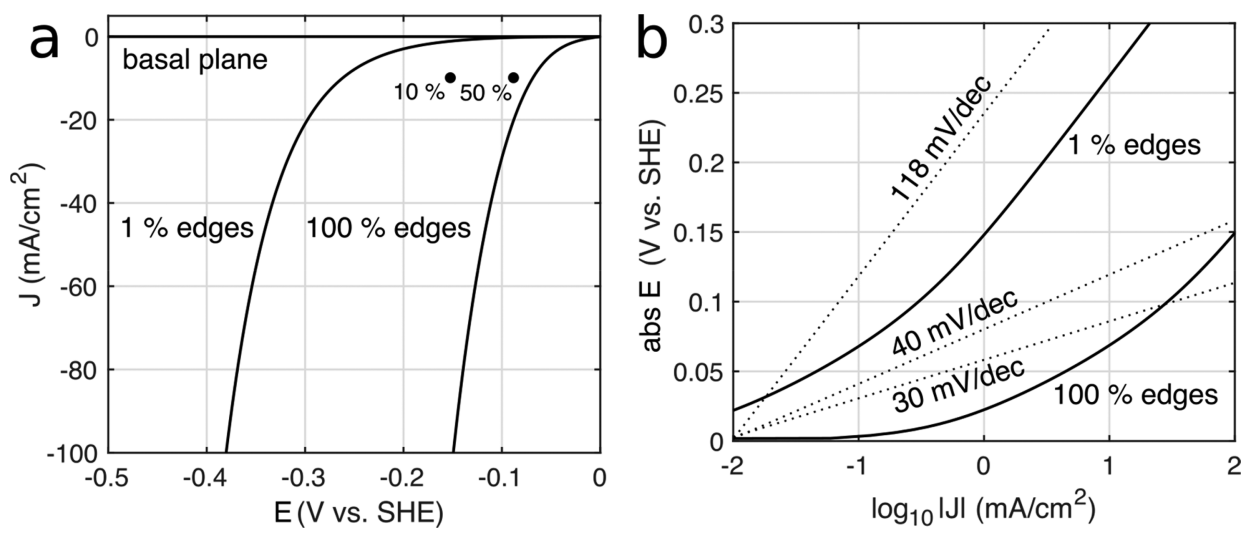

Figure 7. (a) Theoretical polarization curves of three different structures consisting of a $2 \mathrm{H} \mathrm{MoS}_{2}$ basal plane and edge atoms. The two filled circles mark where $10 \mathrm{~mA} / \mathrm{cm}^{2}$ is achieved for structures with 10 and $50 \%$ edges. (b) Tafel plot of the 1 and $100 \%$ edge-containing structures. Tafel lines showing slopes of 30,40, and $118 \mathrm{mV} / \mathrm{dec}$ are shown as dotted lines. The Tafel line from the basal plane is out of range and therefore not shown.

current-density region, a common issue when measuring Tafel slopes experimentally. This is a consequence of having both negative and positive values of $\Delta G_{\mathrm{H}}$.

\section{CONCLUSIONS}

We presented a method to find equilibrium hydrogen coverages and the corresponding $\Delta G_{\mathrm{H}}$ values. These are later fed into a microkinetic model that can be applied to heterogeneous materials containing large variations in $\Delta G_{\mathrm{H}}$. By combining all adsorption sites, the total activity and detailed information on the material can be obtained. We discussed the accuracy of the model and showed that it compares well with most experimental materials at varying overpotentials. We foresee that this methodology can provide detailed HER characteristics for most catalysts, displaying the rates of each elementary reaction step and the hydrogen coverage at varying overpotentials. We have carefully tested several metals using our model with good agreement between theoretical and experimental results. The results of testing heterogeneous $\mathrm{MoS}_{2}$ catalysts containing edges and basal plane active sites agree very well with experimental observations, showing that our model also works for heterogeneous materials, where all adsorption sites can be included.

\section{ASSOCIATED CONTENT}

\section{(s) Supporting Information}

The Supporting Information is available free of charge at https://pubs.acs.org/doi/10.1021/acs.jpcc.0c05243.

Tables S1-S3, Figures S1-S3 (PDF)

MATLAB script and input files (ZIP)

SIESTA script and input files (ZIP)

\section{AUTHOR INFORMATION}

\section{Corresponding Authors}

Eduardo Gracia-Espino - Department of Physics, Umea University, Umea 90187, Sweden; 기 orcid.org/0000-00019239-0541; Email: Eduardo.gracia@umu.se

Thomas Wågberg - Department of Physics, Umea University, Umeå 90187, Sweden; 이이이.org/0000-0002-5080-8273; Email: Thomas.wagberg@umu.se

\footnotetext{
Author

Joakim Ekspong - Department of Physics, Umeå University, Umea 90187, Sweden
}

Complete contact information is available at:

https://pubs.acs.org/10.1021/acs.jpcc.0c05243

\section{Funding}

T.W. acknowledges support from Vetenskapsrådet (201704862) Energimyndigheten (45419-1). E.G.-E. acknowledges support from Vetenskapsradet (2018-03937) and the Olle Engkvist Foundation (186-0637).

\section{Notes}

The authors declare no competing financial interest.

\section{ACKNOWLEDGMENTS}

The theoretical simulations were performed on resources provided by the Swedish National Infrastructure for Computing (SNIC) at the High Performance Computing Center North (HPC2N).

\section{REFERENCES}

(1) Carmo, M.; Fritz, D. L.; Mergel, J.; Stolten, D. A comprehensive review on PEM water electrolysis. Int. J. Hydrogen Energy 2013, 38 (12), 4901-4934.

(2) Dincer, I.; Acar, C. Review and evaluation of hydrogen production methods for better sustainability. Int. J. Hydrogen Energy 2015, 40 (34), 11094-11111.

(3) Ball, M.; Weeda, M. The hydrogen economy - Vision or reality? Int. J. Hydrogen Energy 2015, 40 (25), 7903-7919.

(4) Nikolaidis, P.; Poullikkas, A. A comparative overview of hydrogen production processes. Renewable Sustainable Energy Rev. 2017, 67, 597-611.

(5) Eftekhari, A. Electrocatalysts for hydrogen evolution reaction. Int. J. Hydrogen Energy 2017, 42 (16), 11053-11077.

(6) Zou, X.; Zhang, Y. Noble metal-free hydrogen evolution catalysts for water splitting. Chem. Soc. Rev. 2015, 44 (15), 5148-80.

(7) Norskov, J. K.; Bligaard, T.; Rossmeisl, J.; Christensen, C. H. Towards the computational design of solid catalysts. Nat. Chem. 2009, 1 (1), 37-46.

(8) Seh, Z. W.; Kibsgaard, J.; Dickens, C. F.; Chorkendorff, I.; Norskov, J. K.; Jaramillo, T. F. Combining theory and experiment in electrocatalysis: Insights into materials design. Science 2017, 355 (6321), eaad4998.

(9) Parsons, R. The rate of electrolytic hydrogen evolution and the heat of adsorption of hydrogen. Trans. Faraday Soc. 1958, 54 (0), 1053-1063.

(10) Shinagawa, T.; Garcia-Esparza, A. T.; Takanabe, K. Insight on Tafel slopes from a microkinetic analysis of aqueous electrocatalysis for energy conversion. Sci. Rep. 2015, 5, 13801. 
(11) de Chialvo, M. R. G.; Chialvo, A. C. Hydrogen evolution reaction: Analysis of the Volmer-Heyrovsky-Tafel mechanism with a generalized adsorption model. J. Electroanal. Chem. 1994, 372 (1), 209-223.

(12) Gennero de Chialvo, M. R.; Chialvo, A. C. Kinetics of hydrogen evolution reaction with Frumkin adsorption: re-examination of the Volmer-Heyrovsky and Volmer-Tafel routes. Electrochim. Acta 1998, 44 (5), 841-851.

(13) Vilekar, S. A.; Fishtik, I.; Datta, R. Kinetics of the Hydrogen Electrode Reaction. J. Electrochem. Soc. 2010, 157 (7), B1040.

(14) Saraby-Reintjes, A. The hydrogen evolution reaction under mixed kinetic control. J. Chem. Soc., Faraday Trans. 1 1986, 82 (11), 3343-3355.

(15) Skúlason, E.; Tripkovic, V.; Björketun, M. E.; Gudmundsdóttir, S.; Karlberg, G.; Rossmeisl, J.; Bligaard, T.; Jónsson, H.; Nørskov, J. K. Modeling the Electrochemical Hydrogen Oxidation and Evolution Reactions on the Basis of Density Functional Theory Calculations. J. Phys. Chem. C 2010, 114 (42), 18182-18197.

(16) Nørskov, J. K.; Bligaard, T.; Logadottir, A.; Kitchin, J. R.; Chen, J. G.; Pandelov, S.; Stimming, U. Trends in the Exchange Current for Hydrogen Evolution. J. Electrochem. Soc. 2005, 152 (3), J23.

(17) Skulason, E.; Karlberg, G. S.; Rossmeisl, J.; Bligaard, T.; Greeley, J.; Jonsson, H.; Norskov, J. K. Density functional theory calculations for the hydrogen evolution reaction in an electrochemical double layer on the Pt(111) electrode. Phys. Chem. Chem. Phys. 2007, 9 (25), 3241-50.

(18) Rossmeisl, J.; Skúlason, E.; Björketun, M. E.; Tripkovic, V.; Nørskov, J. K. Modeling the electrified solid-liquid interface. Chem. Phys. Lett. 2008, 466 (1-3), 68-71.

(19) Zeradjanin, A. R.; Grote, J.-P.; Polymeros, G.; Mayrhofer, K. J. J. A Critical Review on Hydrogen Evolution Electrocatalysis: Reexploring the Volcano-relationship. Electroanalysis 2016, 28 (10), 2256-2269.

(20) Schmickler, W.; Trasatti, S. Comment on "Trends in the Exchange Current for Hydrogen Evolution" [J. Electrochem. Soc., 152, J23 (2005)]. J. Electrochem. Soc. 2006, 153 (12), L31.

(21) Foo, K. Y.; Hameed, B. H. Insights into the modeling of adsorption isotherm systems. Chem. Eng. J. 2010, 156 (1), 2-10.

(22) Soler, J. M.; Artacho, E.; Gale, J. D.; García, A.; Junquera, J.; Ordejón, P.; Sánchez-Portal, D. The SIESTA method forab initioorderNmaterials simulation. J. Phys.: Condens. Matter 2002, 14 (11), 27452779.

(23) Hohenberg, P.; Kohn, W. Inhomogeneous Electron Gas. Phys. Rev. 1964, 136 (3B), B864-B871.

(24) Hammer, B.; Hansen, L. B.; Nørskov, J. K. Improved adsorption energetics within density-functional theory using revised PerdewBurke-Ernzerhof functionals. Phys. Rev. B: Condens. Matter Mater. Phys. 1999, 59 (11), 7413-7421.

(25) Junquera, J.; Paz, Ó.; Sánchez-Portal, D.; Artacho, E. Numerical atomic orbitals for linear-scaling calculations. Phys. Rev. B: Condens. Matter Mater. Phys. 2001, 64 (23), 235111.

(26) Monkhorst, H. J.; Pack, J. D. Special points for Brillouin-zone integrations. Phys. Rev. B 1976, 13 (12), 5188-5192.

(27) Greeley, J.; Mavrikakis, M. Surface and Subsurface Hydrogen: Adsorption Properties on Transition Metals and Near-Surface Alloys. J. Phys. Chem. B 2005, 109 (8), 3460-3471.

(28) Zheng, J.; Sheng, W.; Zhuang, Z.; Xu, B.; Yan, Y. Universal dependence of hydrogen oxidation and evolution reaction activity of platinum-group metals on $\mathrm{pH}$ and hydrogen binding energy. Science Advances 2016, 2 (3), e1501602.

(29) Sabatier, P. La Catalyse en Chimie Organique. Librairie Polytechnique: Paris et Liege, 1920.

(30) Langmuir, I. The Adsorption of Gases on Plane Surfaces of Glass, Mica and Platinum. J. Am. Chem. Soc. 1918, 40 (9), 1361-1403.

(31) Marković, N. M.; Grgur, B. N.; Ross, P. N. TemperatureDependent Hydrogen Electrochemistry on Platinum Low-Index Single-Crystal Surfaces in Acid Solutions. The. J. Phys. Chem. B 1997, 101 (27), 5405-5413.

(32) Yang, G.; Akhade, S. A.; Chen, X.; Liu, Y.; Lee, M. S.; Glezakou, V. A.; Rousseau, R.; Lercher, J. A. The Nature of Hydrogen Adsorption on Platinum in the Aqueous Phase. Angew. Chem., Int. Ed. 2019, 58 (11), 3527-3532.

(33) Biegler, T.; Rand, D. A. J.; Woods, R. Limiting oxygen coverage on platinized platinum; Relevance to determination of real platinum area by hydrogen adsorption. J. Electroanal. Chem. Interfacial Electrochem. 1971, 29 (2), 269-277.

(34) Jerkiewicz, G. Hydrogen sorption ATIN electrodes. Prog. Surf. Sci. 1998, 57 (2), 137-186.

(35) Bockris, J. O. M.; Reddy, A. K. N.; Gamboa-Aldeco, M. E. Modern Electrochemistry 2A. 2nd ed.; Springer: 2000; p 763.

(36) Trasatti, S. Work function, electronegativity, and electrochemical behaviour of metals: III. Electrolytic hydrogen evolution in acid solutions. J. Electroanal. Chem. Interfacial Electrochem. 1972, 39 (1), 163-184.

(37) Sun, Y.; Lu, J.; Zhuang, L. Rational determination of exchange current density for hydrogen electrode reactions at carbon-supported Pt catalysts. Electrochim. Acta 2010, 55 (3), 844-850.

(38) Tavares, M. C.; Machado, S. A. S.; Mazo, L. H. Study of hydrogen evolution reaction in acid medium on Pt microelectrodes. Electrochim. Acta 2001, 46 (28), 4359-4369.

(39) Parsons, R. Hydrogen evolution on platinum electrodes. The heats of activation for the component reactions. Trans. Faraday Soc. 1960, $56(0), 1340-1350$.

(40) Parsons, R. Handbook of Electrochemical Constants; Butterworths: London, 1959.

(41) Conway, B. E.; Jerkiewicz, G. Relation of energies and coverages of underpotential and overpotential deposited $\mathrm{H}$ at $\mathrm{Pt}$ and other metals to the 'volcano curve' for cathodic $\mathrm{H} 2$ evolution kinetics. Electrochim. Acta 2000, 45 (25), 4075-4083.

(42) Saji, V. S.; Lee, C. W. Molybdenum, molybdenum oxides, and their electrochemistry. ChemSusChem 2012, 5 (7), 1146-61.

(43) Day, V. W.; Klemperer, W. G. Metal Oxide Chemistry in Solution: The Early Transition Metal Polyoxoanions. Science 1985, 228 (4699), 533.

(44) Jehng, J.-M.; Wachs, I. E. Niobium oxide solution chemistry. J. Raman Spectrosc. 1991, 22 (2), 83-89.

(45) Pentland, N.; Bockris, J. O. M.; Sheldon, E. Hydrogen Evolution Reaction on Copper, Gold, Molybdenum, Palladium, Rhodium, and Iron. J. Electrochem. Soc. 1957, 104 (3), 182.

(46) Lindgren, P.; Kastlunger, G.; Peterson, A. A. A Challenge to the G $\sim 0$ Interpretation of Hydrogen Evolution. ACS Catal. 2020, 10 (1), 121-128.

(47) Martins, M. E.; Zinola, C. F.; Arvia, A. J. Voltammetric response of hydrogen adsorbates on platinum in acid solutions: a possible $\mathrm{H}$ adatom subsurface state. J. Braz. Chem. Soc. 1997, 8, 363-370.

(48) Martins, M. E.; Zinola, C. F.; Andreasen, G.; Salvarezza, R. C.; Arvia, A. J. The possible existence of subsurface $\mathrm{H}$-atom adsorbates and $\mathrm{H} 2$ electrochemical evolution reaction intermediates on platinum in acid solutions. J. Electroanal. Chem. 1998, 445 (1), 135-154.

(49) Frelink, T.; Visscher, W.; van Veen, J. A. R. The third anodic hydrogen peak on platinum; Subsurface H2 adsorption. Electrochim. Acta 1995, 40 (5), 545-549.

(50) Liu, L.; Liu, Y.; Liu, C. Enhancing the Understanding of Hydrogen Evolution and Oxidation Reactions on $\mathrm{Pt}(111)$ through $\mathrm{Ab}$ Initio Simulation of Electrode/Electrolyte Kinetics. J. Am. Chem. Soc. 2020, 142 (11), 4985-4989.

(51) Kibler, L. A.; Hermann, J. M.; Abdelrahman, A.; El-Aziz, A. A.; Jacob, T. New insights on hydrogen evolution at Au single crystal electrodes. Current Opinion in. Electrochemistry 2018, 9, 265-270.

(52) Conway, B. E.; Steacie, E. W. R. Kinetics of electrolytic hydrogen and deuterium evolution. Proc. R. Soc. London, Ser. A 1960, 256 (1284), $128-144$.

(53) Conway, B. E.; Bockris, J. O. M. Electrolytic Hydrogen Evolution Kinetics and Its Relation to the Electronic and Adsorptive Properties of the Metal. J. Chem. Phys. 1957, 26 (3), 532-541.

(54) Fan, L.; Liu, P. F.; Yan, X.; Gu, L.; Yang, Z. Z.; Yang, H. G.; Qiu, S.; Yao, X. Atomically isolated nickel species anchored on graphitized carbon for efficient hydrogen evolution electrocatalysis. Nat. Commun. 2016, 7, 10667. 
(55) El Alami, H.; Creus, J.; Feaugas, X. Influence of the plastic strain on the hydrogen evolution reaction on polycrystalline nickel electrodes in H2S04. Electrochim. Acta 2006, 51 (22), 4716-4727.

(56) Kronberg, R.; Hakala, M.; Holmberg, N.; Laasonen, K. Hydrogen adsorption on MoS2-surfaces: a DFT study on preferential sites and the effect of sulfur and hydrogen coverage. Phys. Chem. Chem. Phys. 2017, 19 (24), 16231-16241.

(57) Jaramillo, T. F.; Jørgensen, K. P.; Bonde, J.; Nielsen, J. H.; Horch, S.; Chorkendorff, I. Identification of Active Edge Sites for Electrochemical H\&lt;sub\&gt;2\&lt;/sub\&gt; Evolution from MoS\&lt;sub\&gt;2\&lt;/sub\&gt; Nanocatalysts. Science 2007, 317 (5834), 100.

(58) Ekspong, J.; Sharifi, T.; Shchukarev, A.; Klechikov, A.; Wågberg, T.; Gracia-Espino, E. Stabilizing Active Edge Sites in Semicrystalline Molybdenum Sulfide by Anchorage on Nitrogen-Doped Carbon Nanotubes for Hydrogen Evolution Reaction. Adv. Funct. Mater. 2016, 26 (37), 6766-6776.

(59) Ekspong, J.; Gracia-Espino, E. Theoretical Analysis of Surface Active Sites in Defective $2 \mathrm{H}$ and $1 \mathrm{~T}^{\prime}$ MoS2 Polymorphs for Hydrogen Evolution Reaction: Quantifying the Total Activity of Point Defects. Advanced Theory and Simulations 2020, 3 (3), 1900213.

(60) Benck, J. D.; Hellstern, T. R.; Kibsgaard, J.; Chakthranont, P.; Jaramillo, T. F. Catalyzing the Hydrogen Evolution Reaction (HER) with Molybdenum Sulfide Nanomaterials. ACS Catal. 2014, 4 (11), 3957-3971.

(61) Zhao, G.; Rui, K.; Dou, S. X.; Sun, W. Heterostructures for Electrochemical Hydrogen Evolution Reaction: A Review. Adv. Funct. Mater. 2018, 28 (43), 1803291.

(62) Voiry, D.; Salehi, M.; Silva, R.; Fujita, T.; Chen, M.; Asefa, T.; Shenoy, V. B.; Eda, G.; Chhowalla, M. Conducting $\operatorname{MoS}(2)$ nanosheets as catalysts for hydrogen evolution reaction. Nano Lett. 2013, 13 (12), 6222-7.

(63) Li, Y.; Wang, H.; Xie, L.; Liang, Y.; Hong, G.; Dai, H. MoS2 nanoparticles grown on graphene: an advanced catalyst for the hydrogen evolution reaction. J. Am. Chem. Soc. 2011, 133 (19), 7296-9. 